\title{
Regulation of renin-angiotensin system in gestational hypertension
}

\author{
Xuan Jin ${ }^{1 \neq * *}$, Jing Zhang ${ }^{1 *}$, Yuanfang Zhu ${ }^{2}$, Pei Li ${ }^{1}$, Guiying Jin ${ }^{2}$ and Yu Zeng ${ }^{3}$ \\ ${ }^{1}$ Center for Assisted Reproduction, the First Affiliated Hospital of Nanchang University, Nanchang, Jiangxi, 330006, China \\ ${ }^{2}$ Department of Obstetrics and Gynecology, the First Affiliated Hospital of Nanchang University, Nanchang, Jiangxi, 330006, China \\ ${ }^{3}$ Department of Surgery, the First Affiliated Hospital of Nanchang University, Nanchang, Jiangxi, 330006, China \\ \#First co-authors contributed equally to the manuscript
}

\begin{abstract}
Background: Hypertension is common disease amongst women with pregnancy. It is associated with significant maternal, fetal and infant morbidity. Current research on the mechanism of the hypertensive disorders is limited. The Renin-Angiotensin System (RAS) is a hormone signaling cascade that regulates blood pressure and systemic electrolyte and fluid balance. Our study was designed to focus on the mechanism of gestational hypertension before preeclampsia occurred, exploring the change in both circulating RAS and local RAS.

Methods: The human kidney tissue were collected from patients suffered renal tumor (n: 5) or chronic renal insufficiency (n: 25). The human placental tissues were collected from woman with normal pregnancies (n: 35) and gestational hypertension (n: 21). There is no significant difference in maternal age between normal and gestational hypertension subjects. All the participants received written information, and signed the consent.

Results: Before delivery, the circulating PRL and Ang II concentration in the gestational hypertension is significantly lower than that in normal pregnancy. There is no significant difference between gestational hypertension and normal pregnancy after delivery. In the local RAS, AT1, Ang II and Ang 1-7 levels in gestational hypertension placenta were significantly higher than normal pregnancy. There is no statistical difference of renin, AGT, and ACE, ACE2 between the two subjects.

Conclusions: The change of Ang (1-7) expression in our study is different from the previous studies in preeclampsia. More attention should be put on the balance of Ang II and Ang (1-7).
\end{abstract}

Abbreviations: RAS: Renin-Angiotensin System; AGT: Angiotensinogen; Ang I: Antiotensin-I; ACE: Angiotensin-Converting Enzyme; Ang II: Angiotensin-II; Ang (1-7): Antiotensin (1-7); CREB: Camp Responsive Element Binding Protein; BP: Blood Pressure; CRI: Chronic Renal Insufficiency; VEGF: Vascular Endothelial Growth Factor; AT1: Angiotensin II Receptor Type 1; PRL: Renin Activity.

\section{Background}

The National High Blood Pressure Education Program of the NHLBI classifies hypertensive disorders of pregnancy into following categories: chronic hypertension, gestational hypertension, preeclampsia, preeclampsia superimposed on preexisting hypertension [1]. Hypertension in pregnancy is defined as $140 / 90 \mathrm{~mm} \mathrm{Hg}$ or greater. Depending on the population studied, the incidence during pregnancy ranges from $0.5 \%$ to $3.0 \%$ [2]. Although the potential harmful effects of elevated blood pressure on both mother and fetus has been concerned, relatively little is known about its pathogenesis and prevention.

Preeclampsia is a disorder of pregnancy characterized by hypertension and proteinuria. Preeclampisa and its convulsive form, eclampisa, are associated with $10 \%$ to $25 \%$ of direct maternal deaths and stillbirths, neonatal morbidity and mortality in low-income and middle-income countries [3]. The Renin-Angiotensin System (RAS) is a hormone signaling cascade that regulates blood pressure and systemic electrolyte and fluid balance. Regulation of the RAS in preeclampisa has been researched, different with uncomplicated pregnancy, in which most circulating RAS components increase, preeclamptic women have lower circulating levels of RAS components [4]. Whether the same case of the level of RAS components in preeclampsia happens in gestational hypertension patients, little study has been set.

Renin is synthesized and released by juxtaglomerular cells of the afferent renal arterioles, and cleaves the 452-amino acid Angiotensinogen (AGT) to yield the 10-amino acid long peptide, antiotensin-I (Ang I). The biologically inactive Ang I is then cleaved by Angiotensin-Converting Enzyme (ACE), made primarily in lung endothelium, to the biologically functional angiotensin-II (Ang II), eight-amino acid long effector molecule of the RAS [5]. The components of RAS are not unique to the kidney but are synthesized in many tissues. Placenta is one of the major local RAS during pregnancy [6]. Delivery of the placenta can usually resolve the acute clinical symptoms of preeclampsia, suggesting that the placenta plays a central

Correspondence to: Xuan Jin, Center for Assisted Reproduction, the First Affiliated Hospital of Nanchang University, Nanchang, Jiangxi, 330006, China, Tel: 86-791-88693296; E-mail: jinxuan725@163.com

Key words: gestational hypertension, pregnancy, renin-angiotensin system (RAS), blood pressure, vascular endothelial growth factor (VEGF), cAMP responsive element binding protein (CREB)

Received: October 16, 2017; Accepted: November 07, 2017; Published: November 11, 2017 
role in preeclampsia pathogenesis. Are the alterations in tissue-specific RAS in placenta same with that detected by plasma components activity? As for local uteroplacental RAS, there are some research have been done, however the results are various and controversial. Shah et al. [7] demonstrated an increase in renin level in the deciduas vera of preeclamptic women. On the contrary, Herse et al. [8] argued that without any increase of other local RAS components observed in decidua of preeclamptic patients except angiotensin receptors AT1R. Anton et al. [9] reports in a clinical trial that AT1R mRNA and Ang II level was significantly higher in preeclampsia patients compared with normal pregnancies, despite a decrease in circulating Ang II. And Ang (1-7) concentrations decreased both in the uterus and placenta in the rat preeclampsia model [10]. Those suggest that it is necessary to conduct further investigation.

The mechanisms that govern the maintenance and reacquisition of the renin phenotype are not well understood. It has been shown that the cAMP pathway is crucial in the regulation of renin synthesis and release [11,12]. Phosphorylated form of cAMP Responsive Element Binding protein (CREB) associated with its co-activators CBP and $\mathrm{p} 300$ mediated the transcriptional effect of cAMP by binding to the cAMP response element present in the upstream region of renin gene [13-16].

Gestational hypertension is elevated blood pressure and without proteinuria, which develops after 20 weeks of gestation in a previously normotensive woman. Approximately $15-45 \%$ gestational hypertension will eventually develop preeclampsia $[17,18]$. Treatment on gestational hypertension can prevent preeclampsia effectively. In the current study we will explore the change in both circulating RAS and local RAS and test whether CREB plays a crutial part on the expression of RAS components including renin during the hypertention in pregnency too.

\section{Materials and methods}

\section{Human subjects}

The human kidney tissue in the experiments were collected from patients suffered renal tumor or chronic renal insufficiency. Subjects (n: 30) aged 40-65 years, were allocated to the study, 17 women and 13 men, 5 of them are suffered renal tumor with normal Blood Pressure (BP), who are designed as control, the rest 25 are suffered Chronic Renal Insufficiency (CRI) with high blood pressure. Tissue samples and blood samples were from these patients. The measurement of blood pressure was conducted by a trained and certified technician. Details of the measurement of blood pressure have been described in ARIC Manual 11: Sitting Blood Pressure and Postural Changes in Blood Pressure and Heart Rate [19].

The human placental tissues in the experiments were collected from woman with normal pregnancies and gestational hypertension, who had either cesarean section or vaginal deliveries. Group of normal pregnancies (n: 35) consisted of normotensive pregnant subjects $(\mathrm{BP}<140 / 90 \mathrm{mmHg}$ ) who have no history of chronic blood pressure elevation and an absence of proteinuria. Group of gestational hypertension (n:21) consisted of pregnant subjects who developed new onset hypertension $(\mathrm{BP}>140 / 90 \mathrm{mmHg}$ ) without proteinuria. Women in both groups were over age 18 years and less than age 45 years, all of them were free of known cardiovascular, renal, cancer, or diabetes. Placental samples were taken after the baby and placenta were delivered.

All the participants received written information, and signed the consent.
The study has been approved by the local medical ethics committee and all the procedures are in accordance with the institutional guidelines.

\section{RNA extraction and Quantitative Real-time PCR}

Tissues were ground in Tri-Reagent (Molecular Research Center, Cincinnati, OH). Total RNA was extracted according to the manufacturer's directions. Contaminating DNA was removed using the DNA-free kit (Ambion, Austin, TX). The cDNA was prepared from 2 $\mu \mathrm{g}$ of RNA using Moloney murine leukemia virus reverse transcriptase (Life Technologies, Grand Island, NY) and an oligo(dT) 15 primer according to the manufacturer's directions. PCR was performed on $2 \mu \mathrm{l}$ of the RT reaction as a template using Taq DNA polymerase (Promega, Madison, WI). The primer sequences for all the genes examined are listed in Table 1.

\section{Immunohistochemistry}

Serial $5 \mu \mathrm{m}$ sections of the tissue were deparaffinized, and rehydrated through degraded ethanol. The sections were incubated with the primary antibodies specific for renin (Santa Cruz), Angiotensin II receptor Type 1 (AT1) (abcam), and CREB (abcam) respectively in 10\% goat serum at room temperature for $2 \mathrm{hr}$, and then biotinylated secondary antibody and horseradish peroxidase-conjugated strepavidin, washing $3 \times 10 \mathrm{~min}$ after each incubating. Normal IgG instead of the primary antibody was served as the negative controls.

\section{Measurement of renin activity (PRL) and antiotensin II (Ang II)}

Circulating renin activity was measured by radioactive immunoassay with quantitative determination of angiotensin I. Blood samples were collected in EDTA containing tubes in ice and centrifuged at 2000x $g$ for 10 minutes, $500 \mu \mathrm{l}$ supernatant of sample were harvested, and added with $10 \mu \mathrm{l}$ phenylmethysulfonyl fluoride and $50 \mu \mathrm{l}$ angiotensin I buffer, after 90 minutes incubation, $50 \mu \mathrm{l}$ sample and $500 \mu$ l tracers were added to the bottom of tubes which were coated with the ${ }^{125} \mathrm{I}$-labeled hormone, BSA, phosphate buffer, stabilizers, preservatives, and an inert red dye. After 3 hours incubation, the mixture was aspirated and a Gamma counter suitable for counting ${ }^{125} \mathrm{I}$ measured the radioactivity of tubes. PRA was calculated as nanograms angiotensin I per milliliter per hour, and Ang II was calculated as picograms Ang II concentration in plasma as per milliliter.

Table 1. Primers used for real-time PCR

\begin{tabular}{|l|l|l|}
\hline & Gene & Primer sequence \\
\hline CREB & Forward & 5' CAAATGGAAAGGAAGGATAATGT 3' \\
\hline & Reverse & 5' CCAAAGAAACAGGAAGCAGATT 3' \\
\hline AGT & Forward & 5' GCTTTCAACACCTACGTCCACT 3' \\
\hline & Reverse & 5' GGCACTTGAGTCACCGAGAA 3' \\
\hline Renin & Forward & 5' GGCTCCTGTACCTTTGGTCT 3' \\
\hline & Reverse & 5' GTGGTGTTGCCAAGTGTCAG 3' \\
\hline ACE & Forward & 5' GGGACTTCTACAACGGCAA 3' \\
\hline & Reverse & 5' CTTGGGCGTAGACACTGAGA 3' \\
\hline ACE2 & Forward & 5' AGGATGTGCGAGTGGCTAAT 3' \\
\hline & Reverse & 5' 'GGCTGTATCCCCAGAAACT 3' \\
\hline AT1 & Forward & 5' GCCCTAAAGAAGGCTTATGAA 3' \\
\hline & Reverse & 5' CAGTCACGTATGATGCCTAGTTG 3' \\
\hline VEGFa & Forward & 5' GAGGGCAGAATCATCACGAA 3' \\
\hline & Reverse & 5' GCACACAGGATGGCTTGAA 3' \\
\hline GAPDH & Forward & 5' AACATACCAGATCCGCACAGCACC 3' \\
\hline & Reverse & 5' GTTCTTCATTAGACGGACCTCTCTCTTCCG 3' \\
\hline & & \\
\hline
\end{tabular}




\section{Western blot}

The tissues from various groups were homogenized in lysis buffer ( $5 \mathrm{mmol} / \mathrm{L}$ phosphate buffer, $\mathrm{pH} 7.2$, containing $0.1 \%$ Triton X-100, 1 $\mathrm{mM}$ phenylmethylsulfonylfluoride, $1 \mathrm{mg} / \mathrm{L}$ chymostatin) respectively and the protein content of the supernatant after centrifugation was determined by spectrophotometer. The sample lysates were mixed with the loading buffer $(62.5 \mathrm{mM}, 1,4$-dithiothreitol, $5 \%$ sodium dodecyl sulfate (SDS), and $10 \%$ glycerol), boiled for $8 \mathrm{~min}$, separated by SDSpolyacrylamide gel electrophoresis $(30 \mu \mathrm{g}$ total protein/lane). After electrophoretic transferred to the polyvinylidene difluoride membrane, the membranes were blocked with 5\% nonfat milk/PBS for $1 \mathrm{~h}$, followed by incubation at $20^{\circ} \mathrm{C}$ for $1 \mathrm{~h}$ with the primary antibodies. Beta-actin or GAPDH was used as a loading control. The membranes were then washed for three times, $5 \mathrm{~min}$ for each, in $5 \%$ milk/PBS and incubated with HRP-conjugated goat anti-rabbit $\operatorname{IgG}(0.04 \mu \mathrm{g} / \mathrm{ml})$ in $5 \%$ milk/ PBS for $1 \mathrm{~h}$ respectively. The membranes were washed in PBS for three times, 5 min for each, followed by 5 min of incubation with SuperSignal West Pico substrate, then subjected to x-ray autoradiography. For the negative controls, the primary antibodies were replaced by the normal IgG of the same origin.

\section{Competitive Enzyme-Linked Immunosorbent Assay (cELISA)}

The specificity of human tissue was tested by an indirect competitive ELISA. 96-well microplates (Nunc MaxiSorp, Roskilde, Denmark) were coated with the respective antigens by adding $100 \mu \mathrm{L}$ of the antigen solution $(2 \mu \mathrm{g} / \mathrm{mL})$ (cloud-clone corp.) in coating buffer $(50 \mathrm{mM}$ sodium carbonate, $\mathrm{pH} 9.5$ ) and incubation overnight at $4{ }^{\circ} \mathrm{C}$. The plates were blocked for $30 \mathrm{~min}$ at room temperature (RT) with $1 \%$ bovine serum albumin (BSA) in PBS and then incubated with either serially diluted tissue sample or undiluted positive and negative controls for 1 $h$ at RT. After three times washing with the provided washing buffer, the plates were incubated with HRP-labeled anti-human IgG (Bio-Rad) 1: 5,000 in PBS-T or for $1 \mathrm{~h}$ at RT. The reaction was stopped by using $50 \mu \mathrm{l}$ of the stopping solution. The immune reaction was detected with TMB ready-to-use substrate (Sigma-Aldrich Co.). The optical density (OD) was measured at $450 \mathrm{~nm}$ in a microtiter plate reader (Tecan, Groedig, Austria). The test samples were considered negative if the optical density $\geq 50 \%$ of the mean of the negative controls.

\section{Statistical analysis}

Values shown are given as the mean \pm SEM. The data were analyzed using one-way ANOVA as appropriate. P-values $<0.05$ were considered statistically significant.

\section{Results}

\section{Blood pressure and plasma RAS components level}

Table 2 shows the clinical profile of the pregnant women, 35 women with normal pregnancies and 21 women with gestational hypertension. The gestational hypertension subjects had hypertension as shown (mean blood pressure: $155 / 96 \mathrm{mmHg}$ versus $128 / 78 \mathrm{mmHg}$ ). None of the subjects had proteinuria. All subjects were matched for gestational age, and there is no significant difference in maternal age between normal and gestational hypertension subjects.

Figure 1 shows the plasma PRL and AngII levels in the normal pregnancies and gestational hypertension. Before delivery, the PRL concentration in the gestational hypertension is significantly lower than that in normal pregnancy. After delivery, concentration of PRL
Table 2. Clinical profile of the pregant women. Values are expressed as means \pm SEMs

\begin{tabular}{|l|l|l|}
\hline $\begin{array}{l}\text { Patient Clinical } \\
\text { Characteristics }\end{array}$ & Normal Pregnancy & Gestational Hypertension \\
\hline $\mathrm{N}$ & 35 & 21 \\
\hline Age (years) & $22.5 \pm 1.5$ & $24.6 \pm 1.2$ \\
\hline Gestational age (weeks) & $37.8 \pm 1.6$ & $37.5 \pm 0.9$ \\
\hline Systolic BP (mm Hg) & $128 \pm 11$ & $155 \pm 8$ \\
\hline Diastolic BP (mm Hg) & $78 \pm 6$ & $96 \pm 4$ \\
\hline Proteinuria & None & None \\
\hline $\begin{array}{l}\text { Number of patients } \\
\text { reveiving } \\
\begin{array}{l}\text { Antihypertensive } \\
\text { medications }\end{array}\end{array}$ & None & None \\
\hline
\end{tabular}

in gestational hypertension increased, and that in normal pregnancy decreased a little, there is no significant difference between gestational hypertension and normal pregnancy after delivery.

For Ang II, it is lower in gestational hypertension before delivery than that in normal pregnancy, and the difference is statistically significant. In the normal pregnancy, Ang II decreased significantly after delivery, there is no statistical difference between normal pregnancy and gestational hypertension after delivery.

\section{RAS components levels in normal and gestational hypertension placenta}

RAS components include renin, Angiotensinogen (AGT), Angiotensin II (Ang II), Angiotensin 1-7 (Ang 1-7), and Angiotensin II receptor (AT), angiotensin-converting enzyme (ACE). The expression of RAS components mRNA and protein was shown in Figure 1. There was no significant difference of mRNA in renin, AGT, AT1, ACE, and ACE2 between normal and hypertension subjects ( $>00.05$ ), although there was trend for higher AT1 mRNA and lower AGT mRNA in gestational hypertension placenta versus normal, however, statistical significance was not reached (Figure 2a). Gestational hypertension placenta was found to have around 4-fold higher AT1 protein compared with normal pregnancy $(0.53 \pm 0.05$ versus $0.14 \pm 0.02$, $\mathrm{P}<0.01$ ) (Figure $2 \mathrm{~b}$ ). In addition, the proteins of Ang II and Ang 1-7 levels in gestational hypertension placenta were significantly higher than the concentrations found in tissue from normal pregnancy (Ang II: $38.15 \pm 5.03$ versus $20.02 \pm 2.07, \mathrm{P}<0.01$; Ang 1-7: $15.44 \pm 2.03$ versus $11.63 \pm 1.83, \mathrm{P}<0.05$ ) (Figure $2 \mathrm{c}$ ). The proteins expression of renin, AGT, and ACE, ACE2 were also determined, no statistical difference was found between the two subjects (Figure 2b).

\section{VEGFa and Renin related gene, CREB expression in placenta and kidney}

Gene expression of VEGFa and CREB in normal pregnant and gestational hypertension placenta was determined by reverse transcription, real-time PCR. No significant difference was found in both of the genes mRNA between the two subjects. In addition, as Figure 4a shown, in normal pregnant placenta versus gestational hypertension, no significant difference was found in the CREB protein expression. Besides that, CREB expression was also detected in human kidney tissues. Human kidney tissues are got from patients suffered with renal tumor or Chronic Renal Insufficiency (CRI). Renal tumor with normal blood pressure is designed as control. A few CREB is found expressed at JG area. Compared with control, there are two results about the level of CREB staining in the CRI kidney tissues, more concentration of CREB immunostaining at JG area in one set (CRI group 1), and similar concentration of CREB expression in the other set (CRI group 
PRL

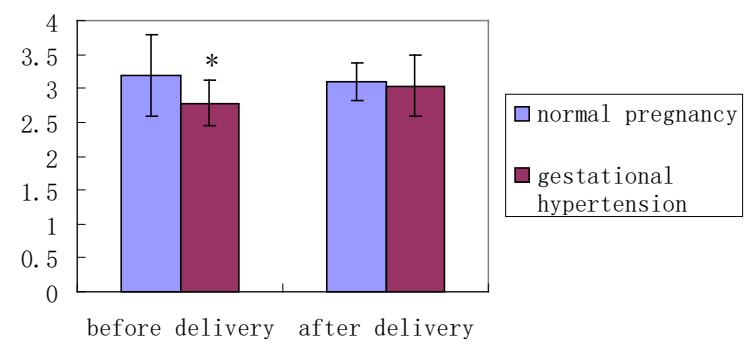

Ang 2

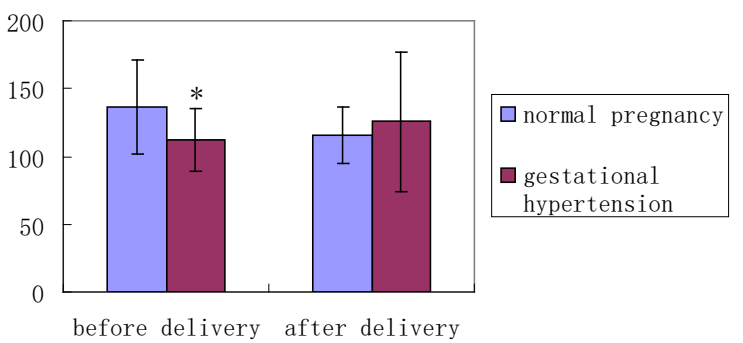

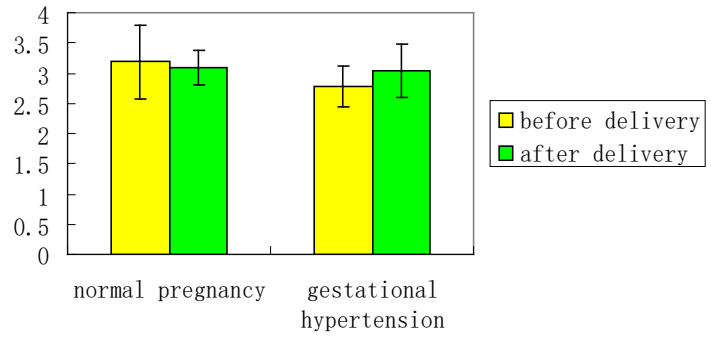

Ang 2

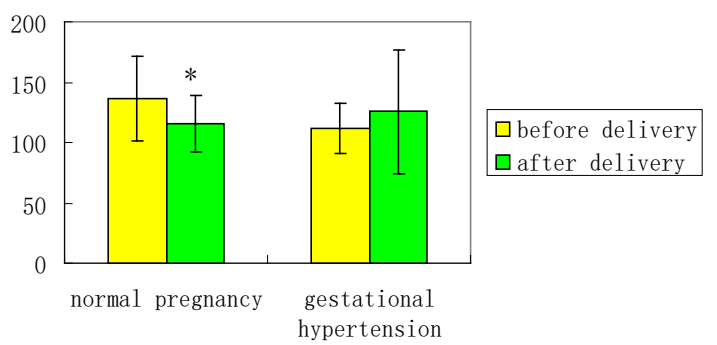

Figure 1. Circulating PRL and AngII levels in normal pregnancy and gestational hypertension . Values are expressed as means $\pm \mathrm{SEMs.} \mathrm{*} \mathrm{P}<0.05$

2) compared with control (Figure $4 \mathrm{~b}$ ). Radioactive immunoassay was used to measure the renin activity in the circulating blood, as figure $2 b$ shown, in the set of high expression of CREB (CRI group 1 ) mean of Plasma Renin Activity (PRA) increased significantly in comparison with the control, same result has been found in Angiotensin II (Ang II), Ang II increased correspondingly in CRI group 1. However, no statistical difference of the level of PRA and AngII has been found between CRI 2 and control. Data of blood pressure has shown that BP is higher than normal standard $(120-140 / 69-90 \mathrm{mmHg})$ in both of the two groups of CRI (Figure 4b).

\section{Discussion}

Hypertension is the most common medical disorder of pregnancy, which includes a spectrum of conditions, most notably preeclampsia, a form of hypertension unique to pregnancy that results in substantial maternal and neonatal morbidity and mortality. The other form, gestational hypertension, usually has more benign courses. Most study has concentrated on preeclampsia and few on gestational hypertension. Our research focus on the mechanism of gestational hypertension before preeclampsia occurred. Blood pressure increasing without proteinuria is the only symptom which happened in the subjects of gestational hypertension.

In an uncomplicated pregnancy, most circulating RAS components increase, however it is not the case in preeclampsia. Preeclamptic women have lower circulating levels of RAS components than their normal pregnant counterparts do [5]. The similar case has been found in gestational hypertension. Before delivery, both circulating active renin (PRL) and Ang II are lower than normotensive pregnant. In addition, we noticed that after delivery, PRL and Ang II decreased in the normal pregnant women, and Ang II has decreased significantly as well, while in the gestational hypertension women, both of PRL and Ang II increased. That can explain partly that no significant difference in PRL and Ang II are detected between normal pregnant and gestational hypertension after delivery.

Since the first-time pro-renin, AGT, ACE, Ang II and Ang I, AT1 were identified in fetal placental tissues in 1998 by Li et al. [20], all the necessary components of RAS have been found both in maternal decidua and fetal placental tissues in succession [21]. However, the levels of RAS components in preeclamptic placental were reported inconsistently $[5,21,22]$. The RAS plays an important role in placental development and regulation of uteroplacental blood circulation. In early pregnancy, Ang II promotes decidualization and rapid trophoblast proliferation mainly by binding to its receptor, AT1 [23]. In mid and late pregnancy, ACE, ACE2 and Ang (1-7) were elevated, which correlate with expanded maternal blood space and progressive fetal capillary development $[10,24,25]$. The dysregulation of RAS in placenta is associated with preeclampsia and other pathophysiological conditions during pregnancy.

Our study is the first to demonstrate the expression of RAS components in human gestational hypertension placental tissues. The classic system involves primarily two enzymes: renin and ACE. Renin mRNA was found to be present in normal and gestational hypertension placenta, besides that, no significant difference of its mRNA and protein levels was found in normal versus gestational hypertension, which is consistent with some previous reports in normal versus preeclamptic [22]. On the contrary, in the study of Singh et al [26] 


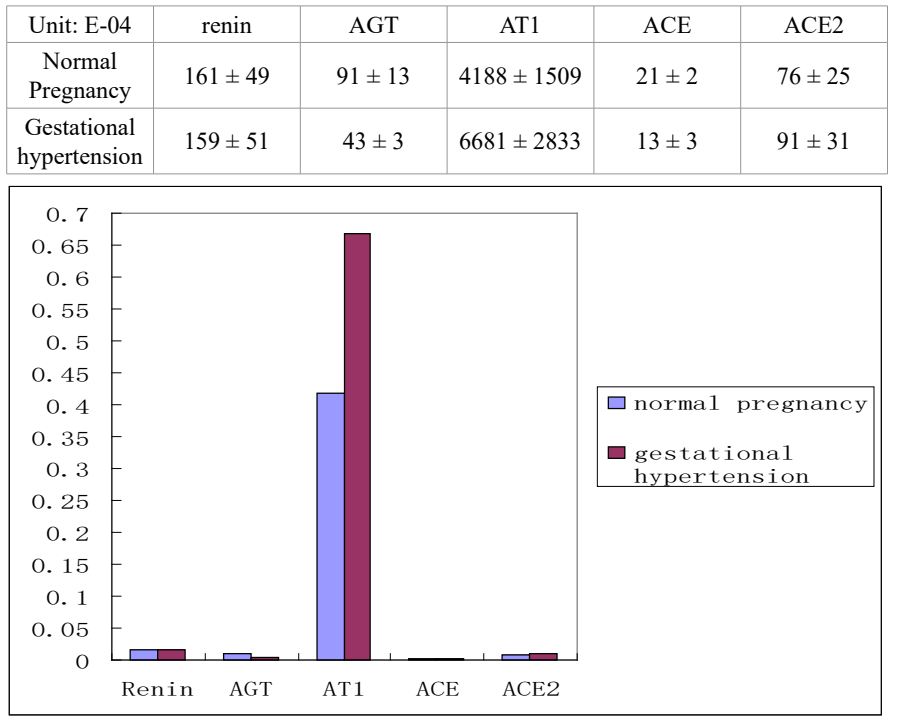

Figure 2a. RAS components mRNA were measured by reverse transcription, real-time PCR of renin, AGT, angiotensin II receptor 1 (AT1), ACE, ACE2.

\begin{tabular}{|c|c|c|c|c|c|}
\hline Unit: E-02 & renin & AGT & AT1 & ACE & ACE2 \\
\hline $\begin{array}{c}\text { Normal } \\
\text { Pregnancy }\end{array}$ & $98 \pm 5$ & $21 \pm 6$ & $14 \pm 2$ & $18 \pm 6$ & $28 \pm 3$ \\
\hline $\begin{array}{c}\text { Gestational } \\
\text { Hypertension }\end{array}$ & $97 \pm 4$ & $26 \pm 7$ & $53 \pm 5$ & $28 \pm 3$ & $30 \pm 7$ \\
\hline
\end{tabular}

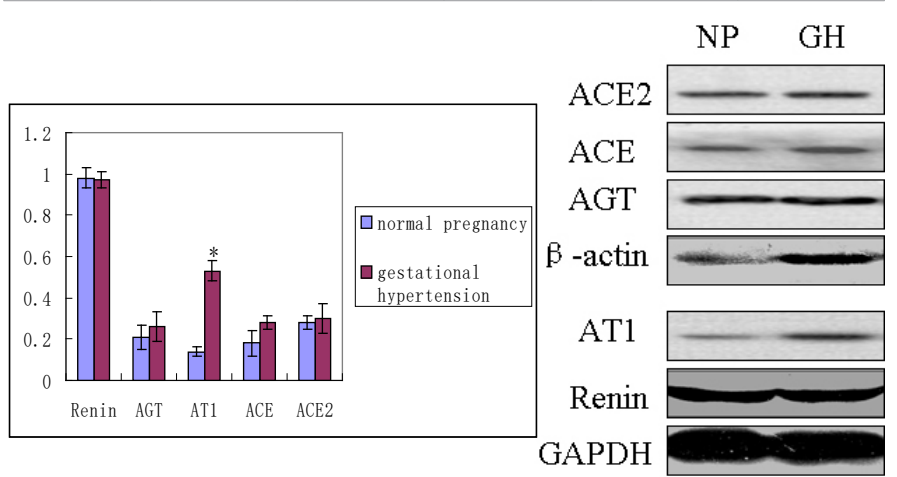

Figure 2b. RAS components protein of renin, AGT, angiotensin II receptor 1 (AT1), ACE, ACE2 were measured by western blot. NP: normal pregnancy GH: gestational hypertension

\begin{tabular}{|c|c|c|}
\hline & Ang 2 & Ang 1-7 \\
\hline Normal Pregnancy & $20.02 \pm 2.07$ & $11.63 \pm 1.83$ \\
\hline Gestational Hypertension & $38.15 \pm 5.03$ & $15.44 \pm 2.03$ \\
\hline
\end{tabular}

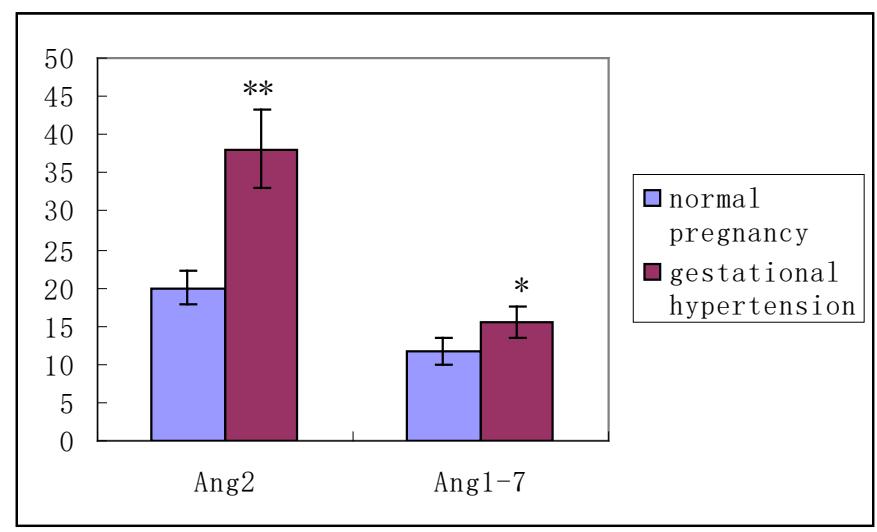

Figure 2c. RAS components protein of Ang 2 and Ang 1-7 were measured by ELISA

Figure 2. Gene expression of RAS components in the placenta of normal pregnancy and gestational hypertension was determined. Values are expressed as means \pm SEMs. * $\mathrm{P}<0.05$, ** $\mathrm{P}<0.01$ 
total renin concentration and active renin were significantly higher in preeclamptic placentas. The different results may indicate a possible trend for increased renin mRNA in the development from gestational hypertension to preeclamptic. ACE functions to hydrolyze Ang I to Ang II, and as its homolog, ACE2 degrade Ang II to Ang (1-7). The interplay between ACE and ACE2 may govern the formation and metabolism of Ang effector peptides. No statistical difference was seen in the levels of ACE and ACE2 between normal and gestational hypertension, which is consistent with most previous studies [8,22,27], except the study of Ito et al, which reported significant increases in ACE mRNA and protein in the preeclamptic placenta. Reasons behind the difference are unclear. The presence of renin, ACE, ACE2 indicates their roles in both normal and gestational hypertension pregnancies.

The rate-limiting step of RAS cascade is the cleavage of AGT by renin to Ang I, a ten-amino acid peptide without any biological function. Then Ang I is cleaved to Ang II, biologically active eight amino acid molecules by ACE. Ang II, binding with its receptor AT1, is regarded as a well-known versatile effector involved in vasoconstriction, sympathetic activity, cellular growth and oxidative stress. Ang II can be cleaved to Ang (1-7) by ACE2. Ang (1-7) is considered to have a counteracting effect to Ang II on the cardiovascular system by its inhibition of cell migration and proliferation [5,21,28]. Expression of AGT was found in our study, and no significant difference was found in the placenta of normal versus gestational hypertension, which was consistent with the study of Herse et al. [8]. However, in the study of Lauren et al, AGT mRNA was higher in the chorionic villi obtained from preeclamptic subjects [22]. The conflicting investigation may result from the tissues studied, what Lauren et al used was exclusively chorinonic villi, while what Herse et al and we used was whole placenta. Another reason that account for the confliction with Lauren et al is our subjects are gestational hypertension, having not developed to preeclampsia yet.

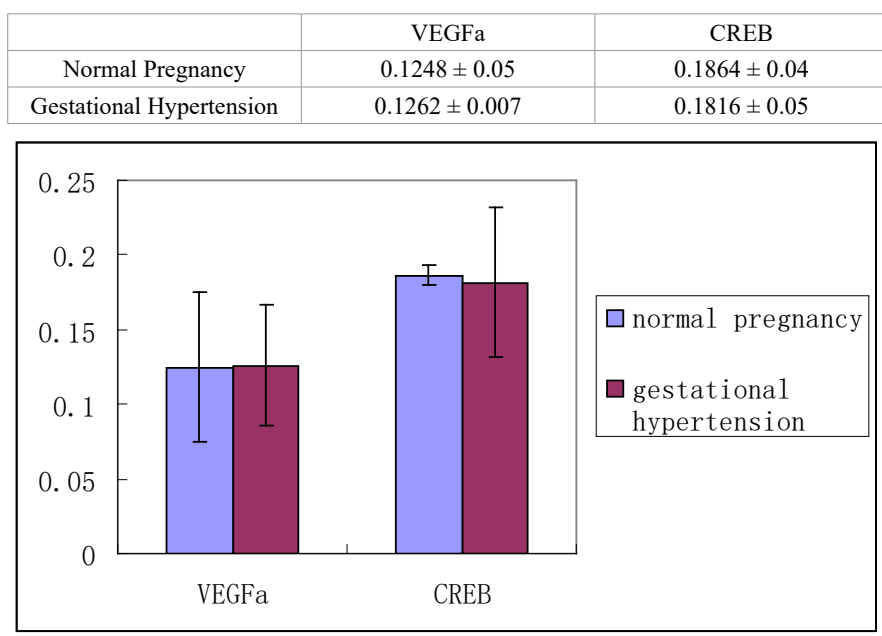

Figure 3. Gene expression of VEGFa and CREB in normal pregnant and gestational hypertension placenta by reverse transcription, real-time PCR

NP

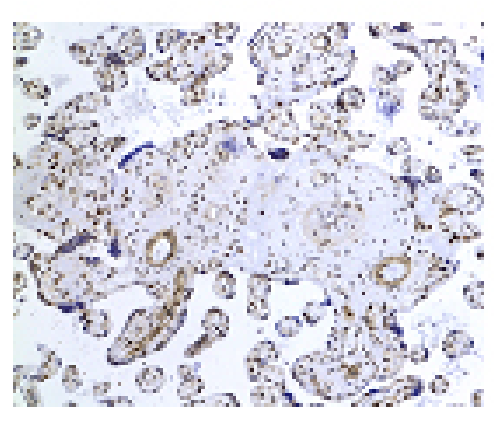

$\mathrm{GH}$

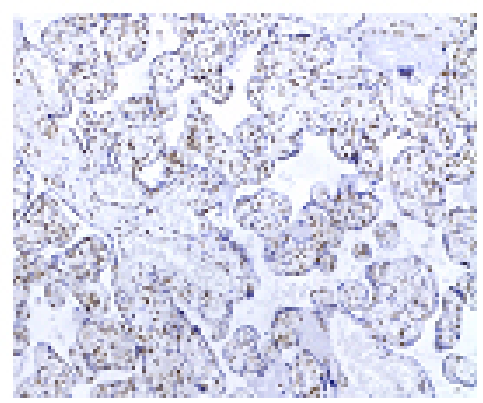

NP GH
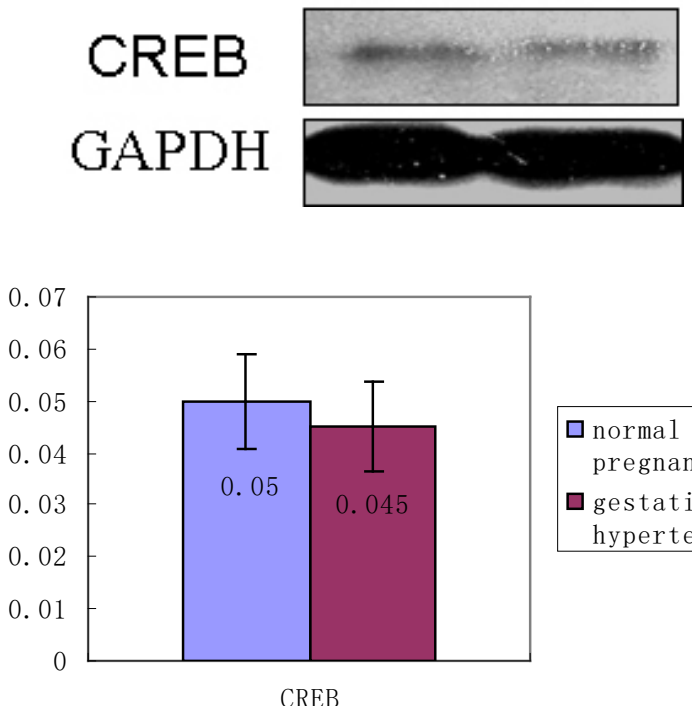

$\square$ normal pregnancy

$\square$ gestational hypertension

Figure 4a. CREB expression detected in normal pregnant and gestational hypertension placenta with Immunohistochemistry and western blot NP: normal pregnancy GH: gestational hypertension 


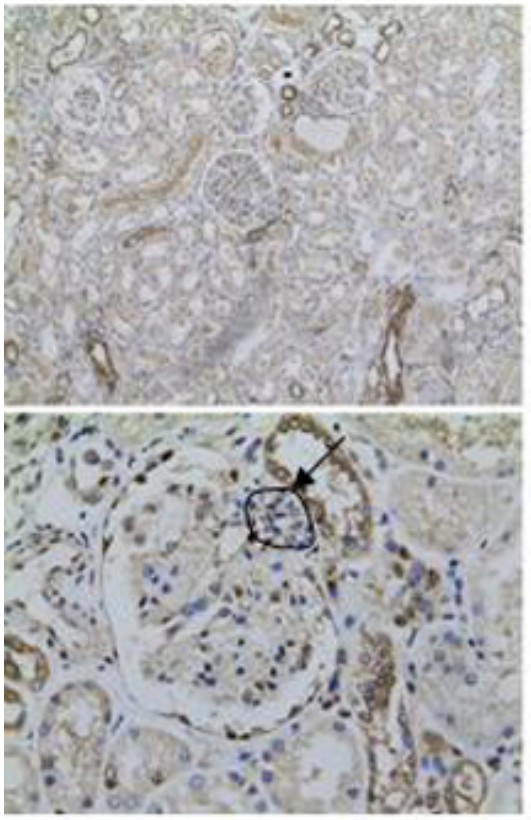

A Renal tumor
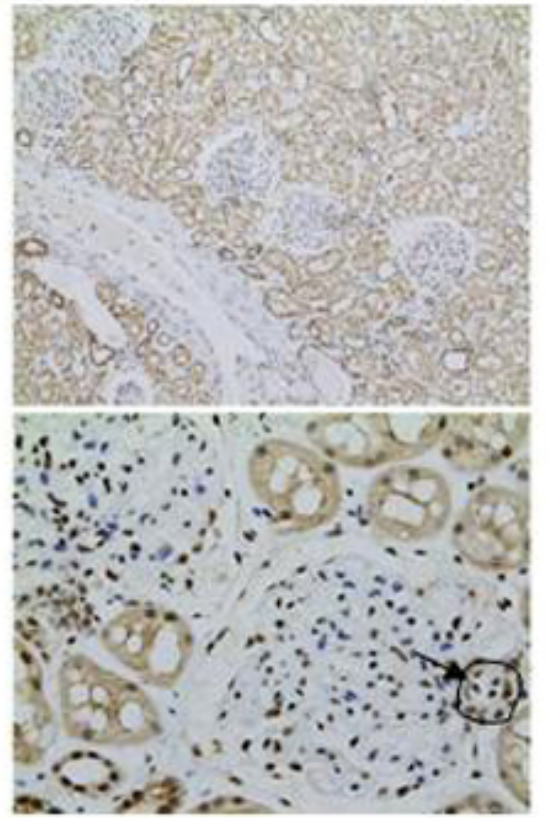

CRI (1)
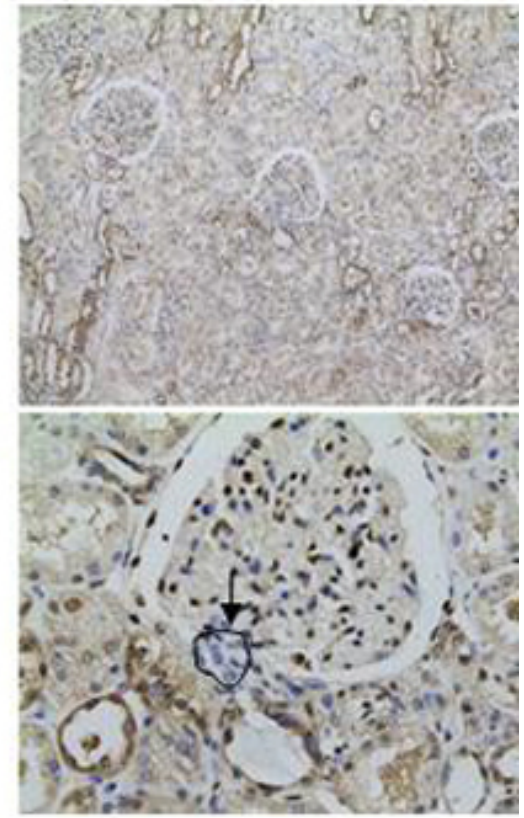

CRI (2)
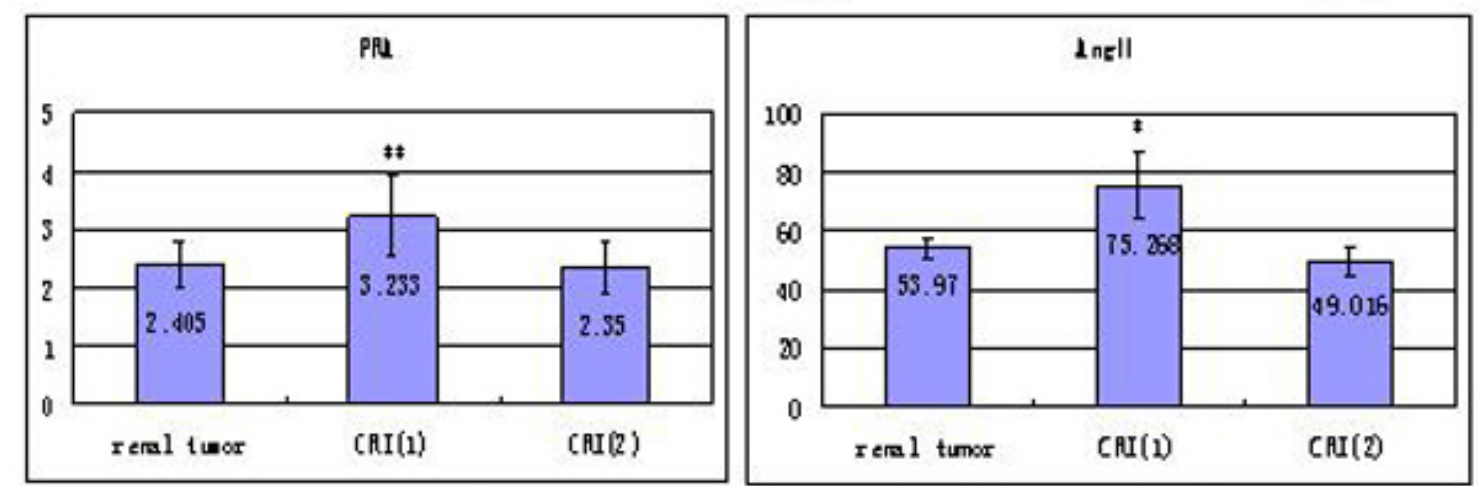

B

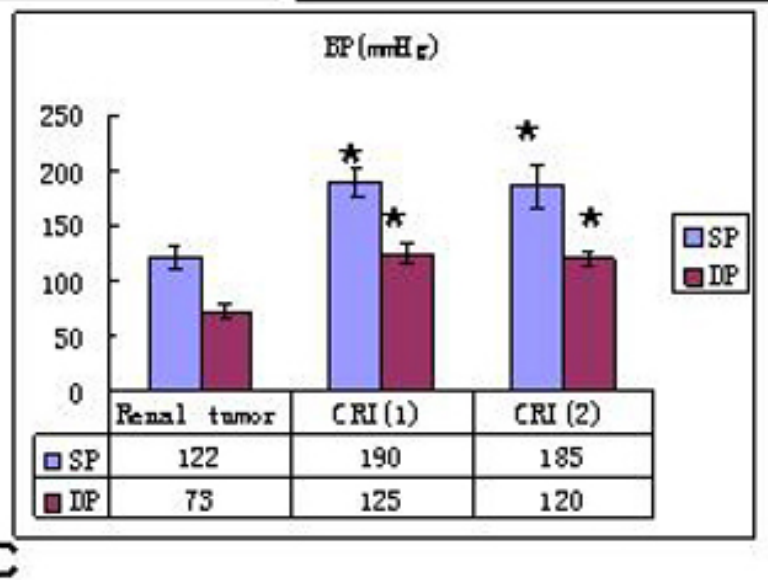

Figure 4b. CREB involved in renin expression in human kidney

A. Immunostaining for CREB in human kidneys from renal tumor, CRI (1) and CRI (2) respectively, figures with high magnification show in JG area more staining of CREB (brown nuclear) in CRI (1) than renal tumor and CRI (2), similar staining of CREB is in the latter two groups. B. Radioactive immunoassay show in the circulating blood renin activity and Ang II of CRI (1) are higher than renal tumor and CRI (2) significantly, no difference of renin activity and Ang II between renal tumor and CRI (2). C. Data of blood pressure show BP of CRI (1) and CRI (2) is higher compared with renal tumor, no difference between CRI (1) and CRI (2), BP of renal tumor is normal. CRI: chronic renal insufficiency PRA: plasma renin activity Ang II: Angiotensin II BP: blood pressure SP: systolic pressure DP: diastolic pressure $* \mathrm{P}<0.05$ vs renal tumor, $* * \mathrm{P}<0.01$ vs renal tumor

Figure 4. Expression of CREB protein in placenta and kidney 
A number of studies have measured the receptor of Ang II, AT1, in the placenta of women with normal and preeclamptic pregnancies, but there is no agreement yet. The conflicting reports include AT1 was upregulated [29], downregulated [30], or not different [8]. It should be noticed that the subjects in these studies are preeclampsia. In our study, the level of AT1 mRNA in the placenta of gestational hypertension is higher than that in normal pregnancy, although there is no statistical difference between them. The level of AT1 protein was found more than 3-fold upregulated in gestational hypertension versus normal. The mismatches of mRNA and protein measurements have been revealed by numerous studies. Consideration should be given to other factors involved in the translation of AT1 gene.

We also found that Ang II was around twice as high in the placenta of gestational hypertension when compared with normal pregnancy, which is same as the result of Lauren [22]. And what interesting is that circulating Ang II was lower in the gestational hypertension. In addition, circulating active renin was lower in the gestational hypertension too, while in the placenta, no difference was found between gestational hypertension and normal. The difference between in circulating and in local placenta suggests redistribution of RAS components, and that contribute to vasoconstriction of the fetal blood vessels and decrease of maternal-fetal transport. Besides that, in contrast with previous studies on the preeclampsia that no difference in Ang (1-7) expression was found versus normal pregnancy [22], or even reduction in Ang (1-7) expression was detected versus normal pregnancy [25,31], we found that in the placenta of gestational hypertension, Ang (1-7) is significantly higher than normal control. It means that at the beginning and benign stage of hypertension during pregnancy, local RAS has been regulated by increasing the expression of Ang (1-7) to try to modulate the effects of RAS on vascular tone, neutralizing excessive contraction. Ang (1-7) counteract the reduction of Ang II in placental blood flow by interacting with AT1. Expression of AT1 protein in the placenta of gestational hypertension is greatly higher than the normal subject can be explained to meet the increasing of Ang II and Ang (1-7). RAS has become a "system within a system" [32,33], Not only ACE-mediated arm of the RAS must be considered, but the ACE2 pathway, giving rise to the vasodilatory and antiproliferative peptide Ang (1-7), should also be put attention to.

It is interesting that in contrast to the significant increase in Ang II and Ang (1-7), no change in ACE and ACE2 levels were found between gestational hypertension and normal control. It suggests that the system is being driven by other regulatory factors. Consideration should also be given to other genes involved in the production of RAS components.

Vascular Endothelial Growth Factor (VEGF) is one of important factors produced from the human placenta, which plays a key role in promoting angiogenesis [34]. Ang II can induce the over expression of VEGF by acting through the AT1 $[35,36]$. While in our study, no difference of VEGFa mRNA was found in gestational hypertension versus normal pregnancy. The result is consistent with previous study in preeclampsia that VEGF release was unchanged by Ang II and Ang (1-7) [37]. The mechanism of angiogenesis in the placenta needs to be explored more.

CREB is known to mediate the stimulation of renin expression, and determine the basal transcription rate of the renin gene [38]. Ang II can lead to the activation of CREB through multiple signaling pathways activating. CREB feed back to upregulate the AT1 gene [39]. In our study on human kidney tissue, we found that the mechanism of all kinds of hypertensive cases is different and complicated. In the chronic renal insufficiency patients, some mechanisms of hypertension are independent of the circulating RAS, and some are caused by renin increased. More CREB was detected at the JG area where renin was expressed in the circulating PRL increased subjects, but not in that no change of PRL subjects. The data shows that renin increased by CREB is one of the causes for high BP in chronic renal insufficiency. However, we did not find any change of CREB expression in the placenta between the gestational hypertension and normal pregnancy, combined with no change of renin expression in placenta either. It seems that regulation on the local RAS in placenta is independent in CREB and renin. It is necessary to conduct further investigation.

\section{Conclusions}

This study provides evidence for the presence and regulation of a local tissue-specific RAS in the benign stage of hypertension during pregnancy, gestational hypertension. Besides of the increase of Ang II and AT1, which is consistent with many other previous reports in preeclampsia, we found that in gestational hypertension local Ang (17) level was significantly higher than normal control. The change of Ang (1-7) expression is different from the studies in preeclampsia. Ang II and Ang (1-7), the two active peptides of the RAS in the placenta, their balance contain the fetal vessels and make up the cell barrier between maternal and fetal blood. The major actions of Ang II may be contributing to the pathophysiology of gestational hypertension, and Ang (1-7) has counteracting effect to Ang II. Increase of Ang (1-7) in the stage of gestational hypertension is the regulation of local RAS, when the balance is broken, the disease would develop into preeclampsia. Because the common factors, CREB and VEGFa, involving in the hypertension and angiogenesis were not found to be the cause for the hypertension during pregnancy, the mechanism of gestational hypertension needs to be investigated more. In summary, hypertension during pregnancy is characterized by a series of significant alterations in RAS worthy of deeper research. The finding of Ang (1-7) increasing in gestational hypertension may lead to a new therapy for diseases.

\section{Authors' contributions}

$\mathrm{XJ}$, as the first and corresponding author, was involved in study design, implementation and execution, data collection and analysis, drafted and coordinated revision of manuscript. JZ, as the first coauthor, was involved in study execution, data analysis, and helped to draft the manuscript. YFZ performed the statistical analysis and communicated with participants. PL, GYJ, and YZ participated in the data acquisition and analysis. All authors read and approved the final manuscript.

\section{Acknowledgement}

All authors read and approved the final manuscript. No conflicts of interest are declared by the authors. The research is supported by National Natural Science Foundation of China 31160246, Natural Science Foundation of Jiangxi province 20114BAB205011, the scientific foundation of health department in Jiangxi province 20121038, and Scientific Grant of Department of Education in Jiangxi Province GJJ13146.

\section{References}

1. Mustafa R, Ahmed S, Gupta A, Venuto RC (2012) A comprehensive review of hypertension in pregnancy. J Pregnancy 2012: 105918. [Crossref]

2. Al-Maawali A, Walfisch A, Koren G (2012) Taking angiotensin-converting enzyme inhibitors during pregnancy: is it safe? Can Fam Physician 58: 49-51. [Crossref]

3. Duley L (2009) The global impact of pre-eclampsia and eclampsia. Semin Perinatol 33: 130-137. [Crossref] 
4. Langer B, Grima M, Coquard C, Bader AM, Schlaeder G, et al. (1998) Plasma active renin, angiotensin $\mathrm{I}$, and angiotensin II during pregnancy and in preeclampsia. Obstet Gynecol 91: 196-202. [Crossref]

5. Roxanna AI, Yang X: Renin angiotensin signaling in normal pregnancy and preeclampsia. Semin Nephrol 2011, 31: 47-58 [Crossref]

6. Shah DM (2006) The role of RAS in the pathogenesis of preeclampsia. Curr Hypertens Rep 8: 144-152. [Crossref]

7. Shah DM, Banu JM, Chirgwin JM, Tekmal RR (2000) Reproductive tissue renin gene expression in preeclampsia. Hypertens Pregnancy 19: 341-351. [Crossref]

8. Herse F, Dechend R, Harsem NK, Wallukat G, Janke J, Qadri F, Hering L, Muller DN, Staff AC: Dysregulation of the circulating and tissue-based rennin-angiotensin system in preeclampsia. Hypertension 2007, 49: 604-611. [Crossref]

9. Anton L, Brosnihan BK: Systemic and uteroplacental rennin-angiotensin system in normal and preeclampsia pregnancies. Ther Adv Cardiovasc Dis 2008, 2: 349-362. [Crossref]

10. Neves LA1, Stovall K, Joyner J, Valdés G, Gallagher PE, et al. (2008) ACE2 and ANG(1-7) in the rat uterus during early and late gestation. Am J Physiol Regul Integr Comp Physiol 294: R151-161 [Crossref]

11. Sequeira Lopez ML, Gomez RA (2010) Novel mechanisms for the control of renin synthesis and release. Curr Hypertens Rep 12: 26-32. [Crossref]

12. Pentz ES, Sequeira Lopez ML, Cordaillat M, Gomez RA: Identity of the renin cell is mediated by cAMP and chromatin remodeling: an in vitro model for studying cell recruitment and plasticity. Am J Physiol Heart Circ Physiol 2008, 294: H699-H707. [Crossref]

13. Arany Z, Newsome D, Oldread E, Livingston DM, Eckner R: A family of transcriptional adaptor proteins targeted by the E1A oncoprotein. Nature 1995, 374: 81-84. [Crossref]

14. Lundblad JR1, Kwok RP, Laurance ME, Harter ML, Goodman RH (1995) Adenoviral E1A-associated protein p300 as a functional homologue of the transcriptional coactivator CBP. Nature 374: 85-88. [Crossref]

15. Gomez RA, Ellen SP, Xuan J, Magali C, Maria LSL (2009) CBP and p300 are essential for renin cell identity and morphological integrity of the kidney. Am J Physiol Heart Circ Physiol 296: 1255-1262. [Crossref]

16. Pan L, Black TA, Shi Q, Jones CA, Petrovic N, et al. (2001) Critical roles of a cyclic AMP responsive element and an E-box in regulation of mouse renin gene expression. $J$ Biol Chem 276: 45530-45538. [Crossref]

17. Taylor RN, Davidge ST, Roberts JM (2009) "Endothelial cell dysfunction and oxidative stress" In: Lindheimer MD, Cunningham FG, Roberts JM (Edr.) Chesley's Hypertensive Disorders in Pregnancy. Elsevier, Amsterdam, The Netherlands, ( $3^{\text {rd }}$ edn $)$ 2009, pp: 145-170. [Crossref]

18. Maynard SE, Min JY, Merchan J, Lim HK, Li J, et al (2003) Excess placental soluble fms-like tyrosine kinase 1 (sFlt 1 ) may contribute to endothelial dysfunction hypertension, and proteinuria in preeclampsia. J Clin Invest 111: 649-658. [Crossref]

19. The ARIC Investigators: ARIC Manual 11, Sitting blood pressure and postural changes in blood pressure and heart rate. ARIC Coordinating Center, Department of Biostatistics, University of North Carolina. Chapel Hill, NC 1987

20. Li X, Shams M, Zhu J, Khalig A, Wilkes M, et al. (1998) Cellular localization of AT1 receptor mRNA and protein in normal placenta and its reduced expression in intrauterine growth restriction. Angiotensin II stimulates the release of vasorelaxants. $J$ Clin Invest 101: 442-454. [Crossref]

21. Jie Y, Jianyu S, Suli Z, Hao L, Huirong L (2013) The role of the renin-angiotensinaldosterone system in preeclampsia: genetic polymorphisms and microRNA. $J \mathrm{Mol}$ Endocrin 50: 53-66. [Crossref]
22. Lauren A, David CM, Liomar AAN, Kathryn S, Patricia EG, et al. (2008) Activation of local chorionic villi antiotensin II levels but not angiotensin (1-7) in preeclampsia. Hypertension, 51: 1066-1072. [Crossref]

23. Hering L, Herse F, Geusens N, Verlohren S, Wenzel K, et al. (2010) Effects of circulating and local uteroplacental angiotensin II in rat pregnancy. Hypertension 56 : 311-318. [Crossref]

24. Levy A, Yagil Y, Bursztyn M, Barkalifa R, Scharf S, Yagil C: ACE2 expression and activity are enhanced during pregnancy. Am J Physiol Regul Integr Comp Physiol 2008, 295: 1953-1961. [Crossref]

25. Haijun G, Uma Y, Chandra Y (2012) Maternal protein restriction reduces expression of angiotensin I-converting enzyme 2 in rat placental labyrinth zone in late pregnancy. Biol Reprod 86: 1-8. [Crossref]

26. Singh HJ, Rahman A, Larmie ET, Nila A: Raised prorenin and renin concentrations in pre-eclamptic placentae when measured after acid activation. Placenta 2004, 25: 631-636. [Crossref]

27. Kalenga MK, Thomas K, deGasparo M, De H: Determination of renin-angiotensin converting enzyme and angiotensin II levels in human placenta, chorion and amnion from women with pregnancy induced hypertension. Clin Endocrinol (Oxf) 1996, 44: 429-433. [Crossref]

28. Wutao Z, Weiyan C, Xiuyu L, Jiangui H, Hong M (2009) Chronic angiotensin-(1-7) administration improves vascular remodeling after angioplasty through the regulation of the TGF- $\beta /$ Smad signaling pathway in rabbits. Biochem Bioph Res Co 389: 138-144.

29. Leung PS, Tsai SJ, Wallukat G, Leung TN, Lau TK (2001) The upregulation of angiotensin II receptor AT (1) in human preeclamptic placenta. Mol Cell Endocrinol 184: 95-102. [Crossref]

30. Knock GA, Sullivan MH, McCarthy A, Elder MG, Polak JM, et al. (1994) Angiotensin II (AT1) vascular binding sites in human placenta from normal-term, preeclamptic and growth retarded pregnancies. J Pharmacol Exp Ther 271: 1007-1015.

31. Merrill DC, Karoly M, Chen K, Ferrario CM, Brosnihan KB (2002) Angiotensin-(1-7) in normal and preeclamptic pregnancy. Endocrine 18: 239-245. [Crossref]

32. Yagil Y, Yagil C (2003) Hypothesis: ACE2 modulates blood pressure in the mammalian organism. Hypertension 41: 871-873. [Crossref]

33. K.Bridget B, Liomar AAN, Mark CC (2005) Does the antiotensin-converting enzyme (ACE)/ACE2 balance contribute to the fate of antiotensin peptides in programmed hypertension? Hypertension 46: 1097-1099. [Crossref]

34. Amaral SL, Linderman JR, Morse MM, Greene AS (2001) Angiogenesis induced by electrical stimulation is mediated by angiotensin II and VEGF. Microcirculation 8: 57-67. [Crossref]

35. Vitoratos N, Hassiakos D, Iavazzo C (2012) Molecular mechanisms of preeclampsia. $J$ Pregnancy 2012: 298343. [Crossref]

36. Vitoratos N, Hassiakos D, Iavazzo C: Molecular mechanisms of preeclampsia. $J$ Pregnancy 2012, article ID: 298343. [Crossref]

37. Lauren A, David CM, Liomar AAN, Courtney G, Cheryl M, Bridget KB (2010) Angiotensin II and angiotensin-(1-7) decrease sFlt1 release in normal but not preeclamptic chorionic villi: an in vitro study. Reprod Biol Endocrinol 8: 135-145. [Crossref]

38. Castrop H, Hocherl K, Kurtz A, Schweda F, Todorov V, et al. (2010) Physiology of kidney renin. Physiol Rev 90: 607-673. [Crossref]

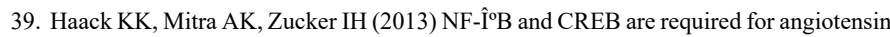
II type 1 receptor upregulation in neurons. PLoS One 8: e78695. [Crossref]

Copyright: (C2017 Jin X. This is an open-access article distributed under the terms of the Creative Commons Attribution License, which permits unrestricted use, distribution, and reproduction in any medium, provided the original author and source are credited. 